\title{
Meta-Analysis of the Efficacy of Non-Steroidal Anti-inflammatory Drugs vs. Opioids for SWL using Modern Electromagnetic Lithotripters
}

\author{
V. A. Mezentsev \\ Harrogate and District NHS Foundation Trust, Yorkshire Deanery, England, United Kingdom
}

\begin{abstract}
Purpose: Clinical studies produce conflicting results on pain relief for shock wave lithotripsy (SWL). We performed a systematic review and meta-analysis to compare non-steroidal anti-inflammatory drugs (NSAIDs) and opioids in pain relief for SWL powered by an electromagnetic generator.

Material and Methods: A search of MEDLINE and EMBASE was performed and all randomized controlled trials comparing NSAIDs and opioids in pain relief for SWL using modern electromagnetic lithotripters were included in the analysis. Data from 3 trials (244 patients) were pooled. The primary outcome measure was adequate analgesia, defined as "if no additional pain relief was used". The difference in the proportion of patients with adequate anesthesia was compared between the NSAIDs and opioids groups as an odds ratio and odds ratio were pooled across the 3 trials with a fixed effects model.

Results: There was no statistically significant difference between using NSAIDs and opioids for pain relief during SWL using modern electromagnetic lithotripters (odds ratio $0.886,95 \% \mathrm{CI} 0.446-1,760, \mathrm{p}=0.730$ ).

Conclusions: Our analysis shows that in relieving pain during SWL using modern electromagnetic lithotripters NSAIDs are as effective as opioids.
\end{abstract}

Key words: extracorporeal shockwave lithotripsy; analgesics; anti-inflammatory agents, non-steroidal; opioids Int Braz J Urol. 2009; 35: 293-8

\section{INTRODUCTION}

The introduction of shock wave lithotripsy (SWL) has radically changed the treatment paradigm for upper urinary tract calculi. Stones that once required an open surgical procedure for effective cure could be treated by SWL in a completely non-invasive manner. When SWL first began in 1980, it was performed with the patient under epidural or general anesthesia (1-5). Modifications in lithotripters over last two decades have made SWL a less painful experience (6). Most current lithotripters are powered by an electromagnetic generator. This allows a wide therapeutic power range (allowing power to be gradually increased) with far less noise for the patient and treating staff. Furthermore, the electromagnetic shock wave source requires little maintenance and has no disposable parts which is commercially attractive. It is generally accepted that pain is caused by the entry of the shock wave at the skin and transmission through deeper structures in addition to the effect on the stone. Many analgesic techniques have been used to treat the sharp, stinging pain produced by the impact of shockwaves. As SWL is performed on a large number of patients, many of whom are treated on an ambulatory basis, the optimal anesthesia technique should be easy to administer, with high efficacy and minimal side effects. The use of opioids is associated with potential complications such as ventilatory depression (7-9), bradycardia (10), hypotension (11), 
nausea, vomiting (7-13) and prolonged recovery time $(12,13)$, prompting the search for a suitable alternative that would provide adequate analgesia with minimal adverse effects. We designed this study to analyze the efficacy of non-steroidal anti-inflammatory drugs (NSAIDs) versus opioids for SWL using modern electromagnetic lithotripters.

\section{MATERIALS AND METHODS}

We searched published reports cited in the Medline and Embase databases between 1988 and 2008 to retrieve fully published English-language clinical studies on pain relief for SWL. In Medline, the search was done by exploding and combining the medical subject heading terms: 'anesthesia' and 'shock wave lithotripsy' and the free text words 'extracorporeal shock wave lithotripsy AND pain relief'. In Embase, the search was done by exploding the ENTREE term: 'shock wave lithotripsy' and combining this with the ENTREE term 'anesthesia' and by using the search term 'extracorporeal shock wave lithotripsy AND pain relief' Studies that analyzed anesthesia for SWL in children were excluded. Reference lists of published articles were also checked to identify relevant studies. Each of the selected articles was reviewed to establish efficacy and side effects of anesthesia for shock wave lithotripsy. Only randomized trials on NSAIDs versus opioids for SWL using lithotripters with the electromagnetic shock wave source were included.

\section{RESULTS}

In all three trials evaluating the efficacy of NSAIDs for extracorporeal shock wave lithotripsy with the electromagnetic shock wave source were selected (13-15). Table-1 provides details of the included trials in terms of the populations studied, the treatment examined and outcome measures used. Key trial characteristics are summarized below.

Two studies used Siemens Lithostar and one trial used Dornier Lithotripter S. All three studies were randomized, although only two trials described the method of randomization. Two studies were double blinded. Only one study reported sample size calculation. The inclusion criteria varied among the trials. All studies included patients older than 15 years old with pelvicalyceal stones.

All studies reported the number of patients who required additional pain relief during SWL as original anesthesia was inadequate. Heterogeneity between studies was not substantial (Table-2). The publication bias in these studies was evaluated graphically with a funnel plot (Figure-1). There was no evidence of publication bias indicated by a lack of asymmetry in the funnel plot. All calculations for meta-analysis for efficacy of non-steroidal anti-inflammatory drugs in SWL were performed using Comprehensive Metaanalysis software Version 2 Biostat, (Englewood, NJ, 2005).

Meta-analysis of randomized studies (total 244 patients) showed that there is no statistically significant difference between using NSAIDs and

Table 1 - Non-steroidal anti-inflammatory drugs (NSAIDS) vs. opioids for pain relief in SWL using the electromagnetic shock wave source.

\begin{tabular}{lccccc}
\hline Study Name & Subgroup within Study & $\begin{array}{c}\text { NSAIDS Adequate } \\
\text { Analgesia } \\
\text { Events }\end{array}$ & $\begin{array}{c}\text { NSAIDS } \\
\text { Total } \\
\text { Number }\end{array}$ & $\begin{array}{c}\text { Opioids } \\
\text { Adequate } \\
\text { Analgesia }\end{array}$ & $\begin{array}{c}\text { Opioids } \\
\text { Total } \\
\text { Number }\end{array}$ \\
\hline Chia YY, 1998 & Tenoxicam vs. fentanyl (A) & 32 & 40 & 28 & 40 \\
Chia YY, 1998 & Tenoxicam vs. tramadol (B) & 32 & 40 & 34 & 40 \\
Yang CP, 2002 & Ketorolac vs. fentanyl & 25 & 30 & 28 & 30 \\
Parkin J, 2002 & Diclofenac vs. alfentanil and diclofenac & 32 & 34 & 30 & 30 \\
\hline
\end{tabular}


Table 2 - Heterogeneity of studies.

\begin{tabular}{lcccc}
\hline Model & \multicolumn{4}{c}{ Heterogeneity } \\
\hline & Q-value & df & p Value & I - squared \\
Fixed & 3.617 & 3 & 0.306 & 17.067 \\
\hline
\end{tabular}

opioids for pain relief during SWL with modern electromagnetic lithotripters lithotripters (odds ratio $0.886,95 \%$ confidence interval: $0.446-1.760, \mathrm{p}=$ 0.730) (Figure-2).

\section{COMMENTS}

Shock wave related pain is an important side effect of SWL and results from stimulation of nociceptive nerve ending in tissues along the shock wave path. There is increasing evidence that the stimulation of nerves by shock waves is not a direct mechanical effect but is mediated by cavitation, the generation and movement of gas bubbles in fluid or tissues (16). Therefore, like many other shock wave effects, pain during shock wave administration could result from cavitation mediated nerve stimulation. There are numerous factors influencing amount of pain during SWL. Apart from patient-related factors, the type of lithotripter, shockwave peak pressure, the size of the

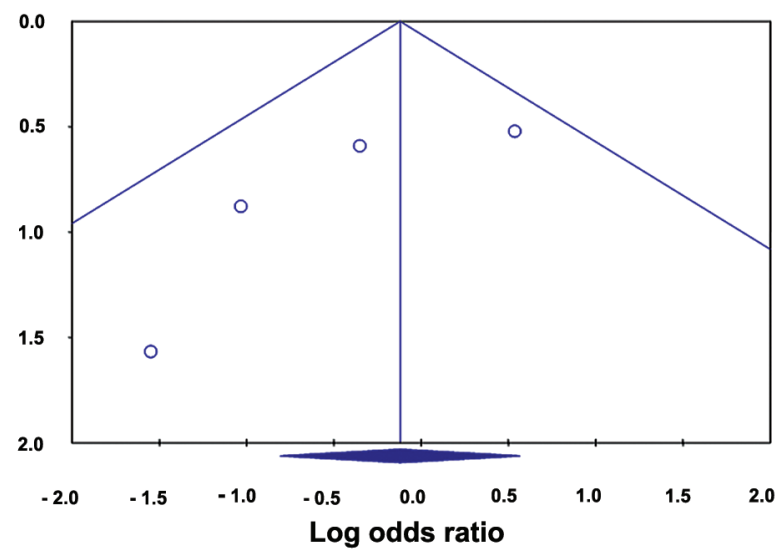

Figure 1 - Funnel plot for randomized studies non-steroidal anti-inflammatory drugs (NSAIDs) vs. opioid. focal zone, and the area of shockwave entry at the skin can change the severity and duration of the pain (17-20). For maximal patient comfort, the most suitable drug for SWL should provide sufficient sedation, adequate analgesia, minimal side effects, and rapid recovery. To the best of our knowledge, to date, no meta-analysis has been carried out to assess pain relief for SWL.

Techniques used to measure pain vary widely from complicated questionnaires such as the McGill questionnaire (21) to the more straightforward the Visual Analogue Scales (VAS). The VAS is usually drawn as a $10 \mathrm{~cm}$ line, with anchors at either end, to depict the extremes of the sensation under study. The subject is asked to mark a point on the scale which indicates to the patient the intensity of the sensation experienced at that time. The only drawback of the VAS is the fact that the patient completes the test after the treatment has been completed. That makes this assessment less reliable than pain assessment during actual treatment. In our analysis we assessed the percentage of patients who requested additional pain relief as original analgesia was inadequate. We feel that those figures are more reliable as they are taken during actual SWL.

Various opioids (morphine, pethidine, tramadol, alfentanil and fentanyl) have been given during SWL using a variety of techniques (bolus subcutaneous/intravenous injections, patient-controlled analgesia) (22). Among the various opioids, fentanyl is a strong synthetic narcotic commonly used during SWL. It has been reported that though fentanyl provided an acceptable analgesia condition in SWL, respiratory depression should be carefully monitored and properly managed. In addition, the personnel who administer opioids should have appropriate reversal agents (e.g., naloxone) on hand in case of severe desaturation. Regarding the risk of desaturation during opioids or sedative administration, continuous noninvasive pulse oximetry should be applied in SWL (15).

Tramadol is a centrally acting analgesic with an unusual mechanism of action involving opioid, noradrenalin and 5-hydroxytryptamine (5-HT) systems (23). It does not result in significant respiratory depression (24). It has been reported that tramadol has caused a high incidence $(25 \%)$ of nausea and vomiting 


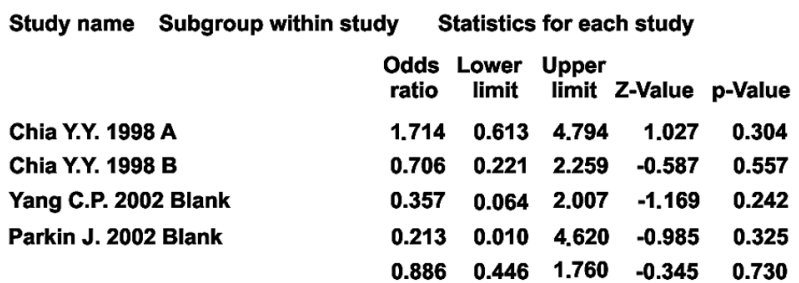

Figure 2 - Meta analysis of non-steroidal anti-inflammatory drugs (NSAIDs) vs. opioid analgesics.
Odds ratio and $95 \% \mathrm{Cl}$

100

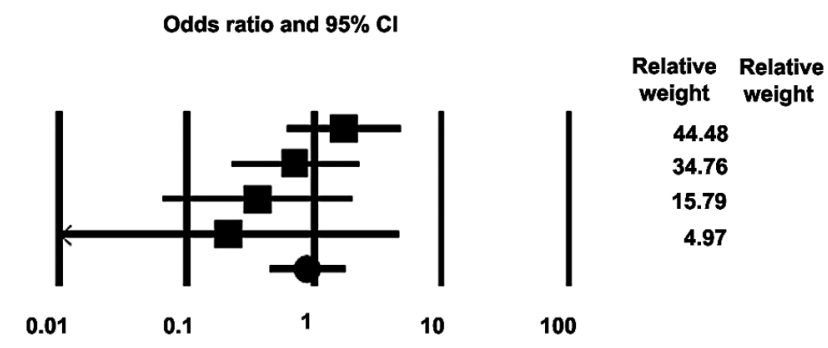

(15). The actual mechanism of nausea and vomiting remains unclear and is assumed to be related to its central effect on opioid receptors.

The NSAIDs, owing their anti-inflammatory effect when given via oral, intramuscular, or rectal use, have been used extensively in SWL. However, side effects may occur, especially affecting the gastrointestinal system, hematopoietic system, and kidneys. NSAIDs reduce the synthesis of cytoprotective prostaglandins by inhibiting cycloxygenase enzyme. They also have antithrombocytic effects because they block the synthesis of thromboxane A2, slow down hemostasis, and may cause a prolonged bleeding time. They reduce renal blood flow, renin release, and the glomerular filtration rate by inhibiting the synthesis of prostaglandin E2 (25). In contrast, NSAIDs have negligible effects on ventilatory control and hemodynamics (17) making it an attractive alternative to opioids for ambulatory surgical setting. The use of opioid analgesics risks respiratory depression especially in susceptible patient groups e.g. the elderly, making the use of NSAID's more attractive - provided there is no contra-indications to their use such as gastrointestinal disturbance, renal dysfunction etc.

Our initial clinical evaluation revealed 46 well designed studies of pain relief in SWL and 33 of those studies were randomized. Only three trials assessed NSAIDs versus opioids using modern electromagnetic lithotripters.

Our analysis shows that in terms of pain relief during SWL using modern electromagnetic lithotripters NSAIDs are as effective as opioids. Provided there are no contraindications to their use, we recommend the use of NSAIDs as a first line of pain relief for SWL powered by an electromagnetic generator.

Several limitations of the source studies, and, by extension, the present meta-analysis merit delineation. The number of studies is small, which limits the ability to evaluate the influence of factors that differ within patient subgroups or across studies. Nonetheless, a meta-analysis of level 1 evidence (randomized controlled trials) is likely minimize bias, as the evidence is evaluated in aggregate. Furthermore, this would reduce the likelihood of random error, as the sample size is greater than that of any of the constituent trials.

\section{CONFLICT OF INTEREST}

None declared.

\section{REFERENCES}

1. Richardson MG, Dooley JW: The effects of general versus epidural anesthesia for outpatient extracorporeal shock wave lithotripsy. Anesth Analg. 1998; 86: 1214-8.

2. Eaton MP, Chhibber AK, Green DR: Subarachnoid sufentanil versus lidocaine spinal anesthesia for extracorporeal shock wave lithotripsy. Reg Anesth. 1997; 22: $515-20$.

3. Lau WC, Green CR, Faerber GJ, Tait AR, Golembiewski JA: Determination of the effective therapeutic dose of intrathecal sufentanil for extracorporeal shock wave lithotripsy. Anesth Analg. 1999; 89: 889-92. 
4. Lau WC, Green CR, Faerber GJ, Tait AR, Golembiewski JA: Intrathecal sufentanil for extracorporeal shock wave lithotripsy provides earlier discharge of the outpatient than intrathecal lidocaine. Anesth Analg. 1997; 84: 1227-31.

5. Coloma M, Chiu JW, White PF, Tongier WK, Duffy LL, Armbruster SC: Fast-tracking after immersion lithotripsy: general anesthesia versus monitored anesthesia care. Anesth Analg. 2000; 91: 92-6.

6. Tritrakarn T, Lertakyamanee J, Koompong P, Soontrapa S, Somprakit P, Tantiwong A, et al.: Both EMLA and placebo cream reduced pain during extracorporeal piezoelectric shock wave lithotripsy with the Piezolith 2300. Anesthesiology. 2000; 92: 1049-54.

7. Gesztesi Z, Rego MM, White PF: The comparative effectiveness of fentanyl and its newer analogs during extracorporeal shock wave lithotripsy under monitored anesthesia care. Anesth Analg. 2000; 90: 567-70.

8. Joo HS, Perks WJ, Kataoka MT, Errett L, Pace K, Honey RJ: A comparison of patient-controlled sedation using either remifentanil or remifentanil-propofol for shock wave lithotripsy. Anesth Analg. 2001; 93: 1227-32.

9. Sa Rego MM, Inagaki Y, White PF: Remifentanil administration during monitored anesthesia care: are intermittent boluses an effective alternative to a continuous infusion? Anesth Analg. 1999; 88: 518-22.

10. Cepeda M.S., Diaz J.E, Hernandez V, Daza E, Carr DB: Music does not reduce alfentanil requirement during patient-controlled analgesia (PCA) use in extracorporeal shock wave lithotripsy for renal stones. J. Pain Symptom Manage. 1998; 16: 382-7

11. Resim S, Gumusalan Y, Ekerbicer HC, Sahin MA, Sahinkanat T: Effectiveness of electro-acupuncture compared to sedo-analgesics in relieving pain during shockwave lithotripsy. Urol Res. 2005; 33: 285-90.

12. Kararmaz A, Kaya S, Karaman H, Turhanoglu S: Effect of the frequency of transcutaneous electrical nerve stimulation on analgesia during extracorporeal shock wave lithotripsy. Urol Res. 2004; 32: 411-5.

13. Yang CP, Cherng CH, Wong CS, Ho ST: Effects of intravenous ketorolac and fentanyl combined with midazolam on analgesia and side effects during extracorporeal shock wave lithotripsy. Acta Anaesthesiol Sin. 2002; 40: 9-12.

14. Parkin J, Keeley FX FX, Timoney AG: Analgesia for shock wave lithotripsy. J Urol. 2002; 167: 1613-5.

15. Chia YY, Liu K: Prospective and randomized trial of intravenous tenoxicam versus fentanyl and tramadol for analgesia in outpatient extracorporeal lithotripsy. Acta Anaesthesiol Sin. 1998; 36: 17-22.
16. Tu J, Matula TJ, Bailey MR, Crum LA: Evaluation of a shock wave induced cavitation activity both in vitro and in vivo. Phys Med Biol. 2007; 52: 5933-44.

17. Dawson C, Vale JA, Corry DA, Cohen NP, Gallagher J, Nockler IB, et al.: Choosing the correct pain relief for extracorporeal lithotripsy. Br J Urol. 1994; 74: 302-7.

18. Ganapathy S, Razvi H, Moote C, Parkin J, Yee I, Gverzdys S, et al.: Eutectic mixture of local anaesthetics is not effective for extracorporeal shock wave lithotripsy. Can J Anaesth. 1996; 43: 1030-4.

19. Tauzin-Fin P, Delort-Laval S, Krol-Houdek MC, Maurette P, Bannwarth B: Effect of balanced analgesia with buprenorphine on pain response and general anaesthesia requirement during lithotripsy procedures. Eur J Anaesthesiol. 1998; 15: 147-52.

20. McDonald PF, Berry AM: Topical anaesthesia for extracorporeal shock wave lithotripsy. Br J Anaesth. 1992; 69: 399-400.

21. Melzack R: The McGill Pain Questionnaire: major properties and scoring methods. Pain. 1975; 1: 277 99.

22. Kumar A, Gupta NP, Hemal AK, Wadhwa P: Comparison of three analgesic regimens for pain control during shockwave lithotripsy using Dornier Delta Compact lithotripter: a randomized clinical trial. J Endourol. 2007; 21: 578-82.

23. Driessen B, Reimann W: Interaction of the central analgesic, tramadol, with the uptake and release of 5-hydroxytryptamine in the rat brain in vitro. $\mathrm{Br} \mathrm{J}$ Pharmacol. 1992; 105: 147-51.

24. Vickers MD, O’Flaherty D, Szekely SM, Read M, Yoshizumi J: Tramadol: pain relief by an opioid without depression of respiration. Anaesthesia. 1992; 47: 291-6.

25. British National Formulary. 2008; 56: 543-51. available from: http://www.bnf.org/bnf

\section{Accepted after revision: January 13, 2009}

\section{Correspondence address:}

Dr. V. A. Mezentsev

Harrogate and District NHS Foundation Trust

Yorkshire Deanery, 19 Old Mill View

Dewsbury, WF12 9QJ, United Kingdom

E-mail: vitalimezentsev@hotmail.com 


\section{EDITORIAL COMMENT}

Extracorporeal shock wave lithotripsy (SWL) is performed throughout the world on a daily basis, but although there are relatively fixed guidelines for the urological management of renal stones, there is a great deal of variation in methods used to manage the pain associated with SWL (1). Different anesthetic techniques including regional, general and local have been used $(1,2)$. Because of the patient discharged home within hours of treatment completion, this has implications in terms of cost effectiveness and efficiency and improving patient acceptance of the treatment (1). Analgesic agent for ambulatory procedures should undergo rapid predictable elimination, produce no toxic metabolites or side effects, be tolerated by all patient populations, and promote rapid recovery (3). Although opioid analgesics are the traditional first line treatment in this setting, they have the potential to cause adverse events, which often leads to reluctance to increase doses to achieve adequate analgesia (4). Extensive use of opioids is associated with a variety of side effects, such as ventilatory depression, drowsiness and sedation, nausea and vomiting, pruritus, urinary retention, which can delay hospital discharge. Therefore, anesthesiologists and surgeons are increasingly turning to non-opioid analgesic techniques as adjuvant for managing pain during the ambulatory procedures to minimize the adverse effects of analgesic medications (5).

As already pointed out by the author, there are many reports suggested that non-steroidal antiinflammatory drugs (NSAIDs) possessed analgesic properties comparable to the traditional opioid analgesics without opioid related side effects. As regards this point, meta-analysis of the efficacy of NSAIDs vs. opioids will be precious in the clinical practice. It would be more interesting if the side effects of the opioids and NSAIDs were documented. The main endpoint of this manuscript was that if there are no contra-indications to their use, NSAIDs might be the first line of pain relief in this ambulatory procedure.

\section{REFERENCES}

1. Medina HJ, Galvin EM, Dirckx M, Banwarie P, Ubben JF, Zijlstra FJ, et al.: Remifentanil as a single drug for extracorporeal shock wave lithotripsy: a comparison of infusion doses in terms of analgesic potency and side effects. Anesth Analg. 2005; 101: 365-70.

2. Richardson MG, Dooley JW: The effects of general versus epidural anesthesia for outpatient extracorporeal shock wave lithotripsy. Anesth Analg. 1998; 86: 1214-8.

3. Nagelhout JJ, Boytim MJ: Pharmacologic rationale for anesthetic agents in ambulatory practice. J Perianesth Nurs. 2001; 16: 371-8.

4. Rosenow DE, Albrechtsen M, Stolke D: A comparison of patient-controlled analgesia with lornoxicam versus morphine in patients undergoing lumbar disk surgery. Anesth Analg. 1998; 86: 1045-50.

5. White PF: The changing role of non-opioid analgesic techniques in the management of postoperative pain. Anesth Analg. 2005; 101 (Suppl 5): S5-22.

Dr. Ayten Bilir Associate Professor Dept of Anaesthesiology \& Reanimation Osmangazi University Medical Faculty Eskisehir, Turkey E-mail:aytbilir@yahoo.com 